Ferrata Storti Foundation

\title{
EVI1 triggers metabolic reprogramming associated with leukemogenesis and increases sensitivity to L-asparaginase
}

Haematologica 2020

Volume 105(8):2118-2129

\section{Correspondence:}

HIROSHI MORITAKE

hiroshi_moritake@med.miyazaki-u.ac.jp

Received: May 2, 2019.

Accepted: October 24, 2019.

Pre-published: October 24, 2019.

doi:10.3324/haematol.2019.225953

Check the online version for the most updated information on this article, online supplements, and information on authorship \& disclosures: www. haematologica.org/content/105/8/2118

(C)2020 Ferrata Storti Foundation

Material published in Haematologica is covered by copyright. All rights are reserved to the Ferrata Storti Foundation. Use of published material is allowed under the following terms and conditions:

https://creativecommons.org/licenses/by-nc/4.0/legalcode. Copies of published material are allowed for personal or internal use. Sharing published material for non-commercial purposes is subject to the following conditions:

https://creativecommons.org/licenses/by-nc/4.0/legalcode, sect. 3. Reproducing and sharing published material for commercial purposes is not allowed without permission in writing from the publisher.

\author{
Yusuke Saito, ${ }^{1}$ Daisuke Sawa, ${ }^{1}$ Mariko Kinoshita, ${ }^{1}$ Ai Yamada, ${ }^{1}$ Sachiyo \\ Kamimura ${ }^{1}$, Akira Suekane, ${ }^{2}$ Honami Ogoh, ${ }^{2}$ Hidemasa Matsuo, ${ }^{3}$ Souichi \\ Adachi, ${ }^{3}$ Takashi Taga, ${ }^{4}$ Daisuke Tomizawa, ${ }^{5}$ Motomi Osato, ${ }^{6,7}$ Tomoyoshi \\ Soga, ${ }^{8}$ Kazuhiro Morishita ${ }^{2}$ and Hiroshi Moritake ${ }^{1}$
}

\begin{abstract}
${ }^{1}$ Division of Pediatrics, Faculty of Medicine, University of Miyazaki, Miyazaki; ${ }^{2}$ Division of Tumor and Cellular Biochemistry, University of Miyazaki, Miyazaki; ${ }^{3}$ Department of Human Health Science, Kyoto University, Kyoto; ${ }^{4}$ Department of Pediatrics, Shiga University of Medical Science, Shiga; ${ }^{5}$ Division of Leukemia and Lymphoma, Children's Cancer Center, National Center for Child Health and Development, Tokyo; ${ }^{6}$ Cancer Science Institute, National University of Singapore, Singapore; 'International Research Center for Medical Sciences, Kumamoto University, Kumamoto and ${ }^{8}$ Institute for Advanced Biosciences, Keio University, Yamagata, Japan
\end{abstract}

\section{ABSTRACT}

M etabolic reprogramming of leukemia cells is important for survival, proliferation, and drug resistance under conditions of metabolic stress in the bone marrow. Deregulation of cellular metabolism, leading to development of leukemia, occurs through abnormally high expression of transcription factors such as MYC and Ecotropic Virus Integration site 1 protein homolog (EVI1). Overexpression of EVI1 in adults and children with mixed lineage leukemia-rearrangement acute myeloid leukemia (MLL-r AML) has a very poor prognosis. To identify a metabolic inhibitor for EVI1-induced metabolic reprogramming in MLL-r AML, we used an XFp extracellular flux analyzer to examine metabolic changes during leukemia development in mouse models of AML expressing MLL-AF9 and Evi1 (Evi1/MF9). Oxidative phosphorylation (OXPHOS) in Evi1/MF9 AML cells accelerated prior to activation of glycolysis, with a higher dependency on glutamine as an energy source. Furthermore, EVI1 played a role in glycolysis as well as driving production of metabolites in the tricarboxylic acid cycle. L-asparaginase (L-asp) exacerbated growth inhibition induced by glutamine starvation and suppressed OXPHOS and proliferation of Evi1/MF9 both in vitro and in vivo; high sensitivity to L-asp was caused by low expression of asparagine synthetase (ASNS) and L-asp-induced suppression of glutamine metabolism. In addition, samples from patients with EVI1+MF9 showed low ASNS expression, suggesting that it is a sensitive marker of Lasp treatment. Clarification of metabolic reprogramming in EVI1'leukemia cells may aid development of treatments for EVI1'MF9 refractory leukemia.

\section{Introduction}

Acute myeloid leukemia (AML) is a cancer of myeloid lineage that results in genetically heterogeneous disease. Chromosomal translocations leading to fusion of the mixed lineage leukemia gene (MLL1) are common alterations associated with aggressive de novo and therapy-related acute leukemia in children and adults. ${ }^{1,2}$

The ecotropic viral integration site-1 (EVI1) gene encodes a zinc finger protein that functions as a transcriptional regulator of hematopoietic stem cell self-renewal and long-term multilineage repopulating activity., ${ }^{3,4}$ Overexpression of EVI1 is an independent, adverse prognostic factor because of its association with reduced remission duration of AML with MLL rearrangement (MLL-r AML), particularly in patients harboring MLL-AF9 (MF9). ${ }^{5.7}$ EVI1 may play an important role in maintenance of cell quiescence and stem cell-like phenotypes in leukemia cells, thereby contributing to chemoresistance. ${ }^{8,9}$ Previously, we identified potential therapeutic 
targets such as CD52 and GPR56 for the treatment of patients with EVI1 ${ }^{\text {high }}$ leukemia; however, these potential targets did not prove effective in practice..$^{10,11}$

Dysregulation of metabolic pathways occurs through aberrant expression of transcription factors such as MYC. ${ }^{12,13}$ Transcriptional changes resulting from aberrantly activated MYC lead to increased glucose uptake and glycolytic activity; it also stimulates glutaminolysis and lipid biosynthesis during leukemogenesis. It is, however, still not known whether MLL positively regulates metabolic pathways. Therefore, we focused on metabolic reprogramming of MF9 AML with high EVI1 expression (EVI1 ${ }^{+}$MF9 AML). A recent study shows that EVI1 alters cellular metabolism and increases activity of CKMT1, which affects mitochondrial respiration and ATP production. ${ }^{14}$ However, further studies are needed to resolve the metabolic relationship between MLL and EVI1..$^{15,16}$

Here, we developed transgenic (TG) mice with high expression of Evil in hematopoietic progenitor cells and established a mouse model of EVI1+MF9 AML. We then examined metabolic changes in these cells during leukemia development using an XFp extracellular flux analyzer. We found that the leukemia cells showed accelerated oxidative phosphorylation (OXPHOS) prior to activation of glycolysis, along with greater dependency on glutamine as an energy source. In addition, treatment with Lasparaginase (L-asp) effectively inhibited proliferation of Evi1'MF9 AML cells by suppressing OXPHOS. Therefore, although patients with EVI1 ${ }^{+}$MF9 AML usually have a poor prognosis, treatment with specific inhibitors of energy metabolic reprogramming induced by EVI1 (e.g. L-asp) may improve their outcomes.

\section{Methods}

\section{TG mice}

The +24 mCNE-mhsp68p vector was kindly provided by Dr. Motomi Osato (Kumamoto University, Kumamoto, Japan). ${ }^{17}$ $+24 \mathrm{mCNE}$ is a stem cell-specific enhancer of Runx1, which is located $+24 \mathrm{~Kb}$ downstream from the transcription start site in mice. Therefore, it is also called eR1 $(+24 \mathrm{~m})$ and is referred to hereafter as eR1. The mEvi1 transgenic construct was engineered by introducing mouse Evi1 cDNA into the EcoR1 site of the eR1mhsp68p vector. A $7.67 \mathrm{~kb}$ Sap1/BsaA1 fragment of eR1-mhsp68p mEvi1 was then microinjected into fertilized mouse eggs (C57BL/6), and TG offspring were screened by polymerase chain reaction (PCR) using the following primers: $5^{\prime}$-ggc cac cat ggc gta tta gg- $3^{\prime}$ and $5^{\prime}$-tct tcc agc gga tag aat gg- $3^{\prime}$. TG mice were backcrossed onto a C57BL/ 6 background and maintained as heterozygotes. All procedures were approved by the University of Miyazaki Animal Care and Use Committee.

\section{Retroviral bone marrow transduction assays}

The pMIG-FLAG-MLL-AF9 plasmid was a kind gift from Daisuke Nakada (Addgene plasmid \# 71443). ${ }^{18}$ Plat-E cells were transiently transfected with MSCV vectors harboring the pCL-Eco plasmid using polyethylenimine (PEI). At four days post injection of wild-type (WT) and Evi1-TG mice with 5-fluorouracil (5-FU) (150 mg/kg; Kyowa Kirin), bone marrow (BM) cells were harvested and incubated for 24 hours (h) in X-Vivo 15 (Lonza, Allendale, NJ, USA) supplemented with $50 \mathrm{ng} / \mathrm{mL}$ SCF, $50 \mathrm{ng} / \mathrm{mL}$ TPO, 10 ng/ mL IL-3, and 10 ng/ mL IL-6 (all from Peprotech, Rocky Hill, NJ, USA). After incubation, cells in retronectin (Clontech, Mountain View, CA, USA)-coated plates were spin-infected
[490 g for 45 minutes $(\mathrm{min})$ at $20^{\circ} \mathrm{C}$ ] with retroviral supernatant supplemented with polybrene $(8 \mu \mathrm{g} / \mathrm{mL})$. BM cells $(500,000)$ were then transplanted into lethally irradiated C57BL/ 6 mice. For secondary transplantations, $\mathrm{GFP}^{+}$cells from primary recipient mice were transplanted into sublethally irradiated recipients.

\section{Measurement of oxygen consumption rate}

Oxygen consumption rate (OCR) was measured using an XFp extracellular flux analyzer (Agilent Technologies, Santa Clara, CA, USA). Leukemia cells were suspended in XF Assay Medium supplemented with $10 \mathrm{mM}$ glucose, $1 \mathrm{mM}$ pyruvate, and $2 \mathrm{mM}$ glutamine. Cells (200,000 per well) were seeded in a culture plate precoated with Cell-Tak (Fisher Scientific). The plate was centrifuged and left to equilibrate for $60 \mathrm{~min}$ in a $\mathrm{CO}_{2}$-free incubator before being transferred to the XFp extracellular flux analyzer. The Mito Stress Test was performed as follows: 1) basal respiration was measured in XF Base Medium (1 mM sodium pyruvate, $10 \mathrm{mM}$ glucose, $2 \mathrm{mM}$ glutamine); 2) oligomycin (1 $\mu \mathrm{M})$ was injected to measure respiration linked to ATP production; 3) the uncoupler carbonyl cyanide 4-(trifluoromethoxy) phenylhydrazone (FCCP, $1 \mu \mathrm{M}$ ) was added to measure maximal respiration; and 4) rotenone and antimycin $\mathrm{A}(0.5 \mu \mathrm{M}$ each) were applied together to measure non-mitochondrial respiration. Measurement of fuel dependency and fuel flexibility was performed according to the manufacturer's Mito fuel flex test kit protocol (Agilent Technologies).

\section{Statistical analysis}

Statistical analysis was performed using a paired Student's $t$-test, the Mann-Whitney U test, or the log-rank test as appropriate. Calculations were performed using Prism software (GraphPad). Group data are expressed as the mean \pm standard deviation. No mice were excluded from data analysis.

\section{Results}

EVI1 induces aggressive MLL-AF9 leukemia to activate mitochondrial respiration

Initially, we created a series of TG mice with high Evil expression (Evi1-TG) in lineage ${ }^{\text {low }} \mathrm{Sca}-1^{+} \mathrm{c}-\mathrm{kit}^{+}$(LSK) and granulocyte/macrophage progenitor (GMP) cells (Figure 1A) under the control of the eR1 enhancer, which shows hematopoietic-specific enhancer activity in both zebrafish and mouse models (Online Supplementary Figure S1A). ${ }^{17}$ There were no differences in blood cell counts or the percentage of normal hematopoietic progenitors including hematopoietic stem cells (HSC), multipotent progenitors (MPP), and GMP between WT and Evil-TG mice (Online Supplementary Figure S1B and C). Evi1-TG mice do not develop leukemia and hematopoietic disorders until at least 16 weeks.

To examine whether EVI1 regulates energy metabolism in MLL-rearranged leukemia cells, we used a murine model in which AML is driven by the MF9 oncogene..$^{19,20}$ Murine hematopoietic progenitor cells from WT and Evi1TG were transduced with retrovirus encoding both MF9 and GFP, and serial colony forming assays were performed. BM cells showing MF9 expression were transplanted into irradiated syngeneic mice (Figure 1B). The colony assay revealed that the colony numbers and percentages of $\mathrm{GFP}^{+}$AML cells from Evi1-TG were higher than those from WT in vitro (Figure $1 \mathrm{C}$ and D). After establishing primary MF9-induced AML in WT or Evi1-TG (WT/MF9 or Evi1/MF9) mice, we noted significantly higher expression of Evi1 in $\mathrm{GFP}^{+}$whole leukemia cells and in 
A

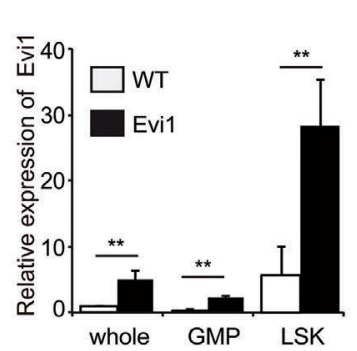

C

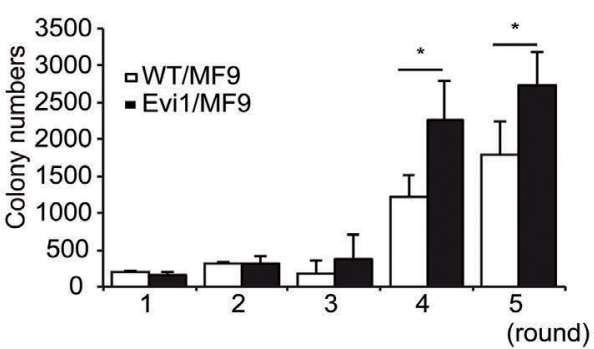

B 5-FU

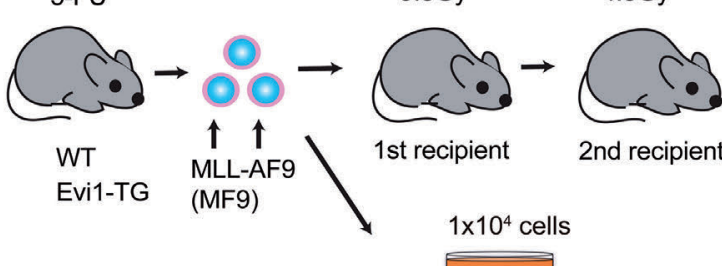

Serial colony forming assay

D

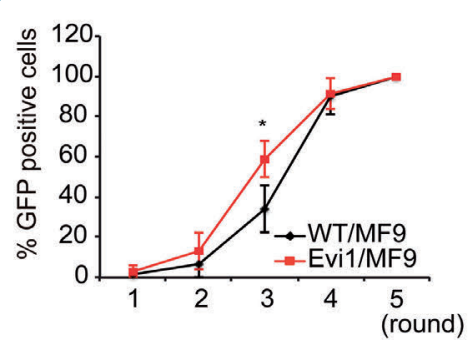

E

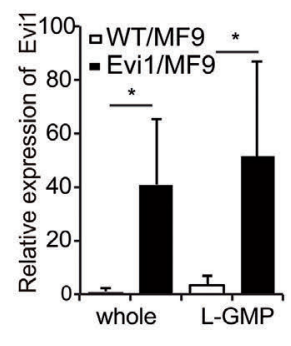

F

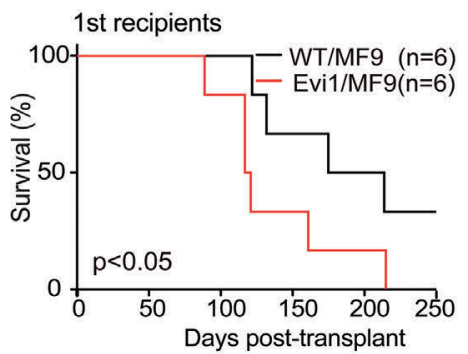

H

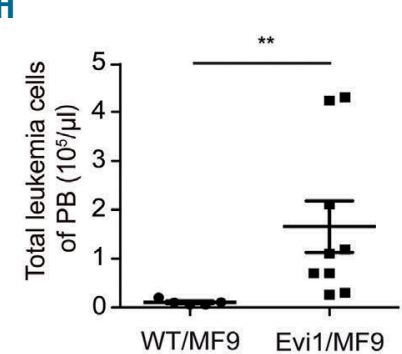

L WT/MF9

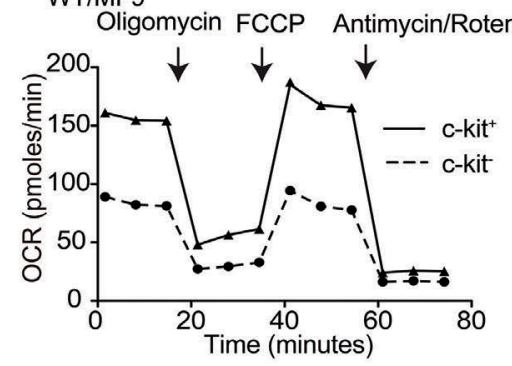

G 2nd recipients $1 \times 10^{4}$
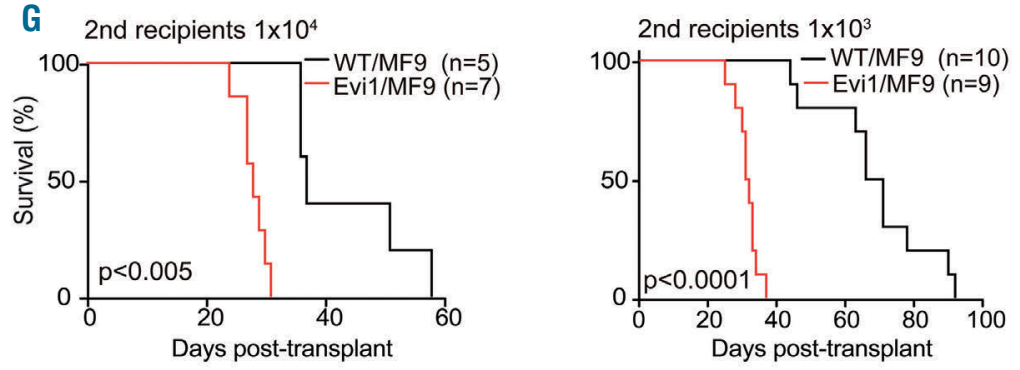

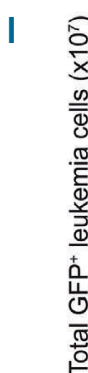

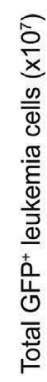

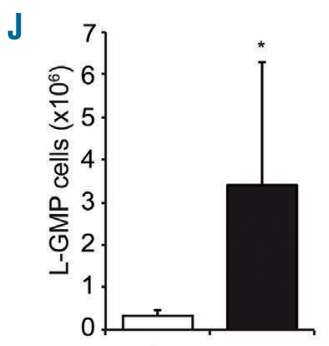

WT/MF9 Evi1/MF9

M Evi1/MF9

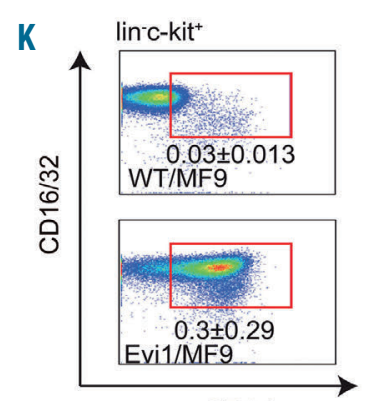

CD34

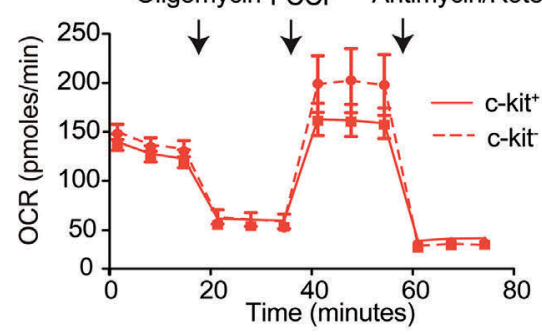

Figure 1. EVI1 induces aggressive MLL-AF9 leukemia cells to activate mitochondrial respiration. (A) Evi1 expression was detected in the LSK fraction, the GMP fraction, and whole bone marrow cells (BM) from wild-type (WT) (white bar) and Evi1-TG mice (black bar) by quantitative real-time polymerase chain reaction (qRT-PCR). Relative expression of Evi1 was normalized against that of $\beta$-actin on whole BM cells. (B) Schematic outline of the mouse model of WT/MF9 and Evi1/MF9. The experimental details are described in the Methods. (C and D) Hematopoietic progenitors from WT (white bar) and Evi1-TG (black bar) mice infected with an MF9 retrovirus were cultured in methylcellulose medium and replated every five days. The colony numbers for each round of replating are indicated in (C). The percentage of GFP-positive cells for each replating round is presented as a line graph (D). (E) Relative expression of Evi1 in the GFP ${ }^{+}$and L-GMP fractions from WT/MF9 or Evi1/MF9 mice, as measured by qRT-PCR. Relative expression of Evi1 was normalized against that of $\beta$-actin on GFP+ cells from WT/MA9. (F) Kaplan-Meier survival curves of mice transplanted with MLL-AF9-transduced WT $(n=6)$ or Evi1-TG cells ( $n=6$ mice; three independent experiments). (G) Secondary transplantation of $10^{4}$ or $10^{3}$ GFP AML cells revealed increased leukemogenesis by Evi1-TG cells ( $n=5-10$ mice; three independent experiments). (H) Total number of GFP ${ }^{+}$leukemia cells in peripheral blood from secondary recipients. (I and J) Total number of acute myeloid leukemia (AML) cells and L-GMP cells in the BM of secondary recipients. Secondary recipients of Evi1/MF9 had significantly more L-GMP in the BM. (K) Representative FACS profiles (gated on lin-c-kit ${ }^{+}$cells) show reduced numbers of CD34 ${ }^{+}$CD16/32 ${ }^{+} L_{-}^{-}$ GMP (red box). ( $\mathrm{L}$ and M). Oxygen consumption rate (OCR) of the c-kit ${ }^{+}$or c-kit- fractions from WT/MF9 (L) and Evi1/MF9 (M). Mitochondrial respiration in c-kit WT/MF9 cells was higher than that in c-kit- cells. By contrast, there was no difference between c-kit ${ }^{+}$and c-kit- cells from Evi1/MF9 mice. All data are expressed as the mean \pm standard deviation. $* P<0.05 ; * * P<0.005 ; * * * P<0.0005$ (Student's $t$-test). Survival curves were compared using the log-rank test. 
the leukemic granulocyte/macrophage progenitor (L-GMP) fraction from Evi1/MF9-recipients (Figure 1E). After the first round of transplantation of BM cells from WT/MF9 or Evi1/MF9, recipient mice receiving Evi1/MF9 cells survived for a significantly shorter time than mice transplanted with WT/MF9 cells (Figure 1F). After a second round of transplantation with $10^{4}$ or $10^{3}$ leukemia cells isolated from primary recipient mice, the survival of secondary recipient mice transplanted with Evi1/MF9 cells was significantly shorter than that of those transplanted with WT/MF9 (Figure 1G). Recipients of Evi1/MF9 cells harbored an increased total number of blast cells in peripheral blood and in the L-GMP fraction in the BM, although there was no difference between these mice and WT/MF9 mice with respect to the total number of $\mathrm{GFP}^{+}$ cells in the BM (Figure 1H-K). A recent report shows that mitochondrial respiration in leukemic stem and progenitor cells is higher than that in differentiated leukemia cells. ${ }^{21,22}$ Therefore, to examine the metabolic advantages conferred by Evi1/MF9 AML cells, we measured the mitochondrial OCR of the stem cell-enriched c-kit-positive $\left(c-k i t^{+}\right)$and differentiated c-kit-negative (c-kit ${ }^{-}$fractions from WT/MF9 or Evi1/MF9 mice. The basal and maximum OCR of c-kit cells from WT/MF9 mice was higher than that of c-kit cells (Figure 1L). However, there was no difference in the OCR between c-kit ${ }^{+}$and c-kit cells from Evi1/MF9 mice (Figure 1M). These data indicate that EVI1 activates mitochondrial respiration in differentiated leukemia cells as efficiently as in the stem cell-enriched fraction, thereby providing a metabolic advantage.

\section{Oxidative phosphorylation is activated before enhanced glycolysis during development of EVI1 ${ }^{+}$ MLL-AF9 leukemia}

To examine the potential role of EVI1 during development of MF9 leukemia, we measured mitochondrial function (i.e. OCR) and glycolytic activity (i.e. ECAR) of serially replated colonies of WT/MF9 and Evi1/MF9 leukemia cells at different replating times using a XFp extracellular flux analyzer. Basal OCR and maximal respiratory capacity in normal c-kit ${ }^{+}$hematopoietic progenitor cells from Evi1-TG mice were lower than in those from WT mice (Figure 2A). However, after the second round of plating, the OCR of Evi1/MF9 progenitor cells at each replating time was higher than that of WT/MF9 cells (Figure 2B-E). To clarify whether mitochondrial respiration is activated by the metabolic environment in BM, we measured OCR in ex vivo WT/MF9 and Evi1/MF9 leukemia cells isolated from the BM of leukemic mice. Evi1/MF9 mice showed a significantly higher basal and maximal respiratory capacity of OCR than WT/MF9 mice (Figure 2F). Of note, the glycolytic activity of Evi1/MF9 cells increased after the $3^{\text {rd }}$ plating (Figure $2 \mathrm{G}$ ); in addition, there was no difference between ex vivo ECAR of WT/MF9 leukemia cells and that of Evi1/MF9 cells (Figure $2 \mathrm{H}$ ). These data suggest that EVI1 activates both OXPHOS and glycolysis; therefore, mitochondrial respiration may play an important role in development of MF9 leukemia and correlate with biological aggressiveness.

\section{EVI1 expression is associated with metabolic reprograming}

To identify the metabolic pathways regulated by EVI1, we performed capillary electrophoresis time-of-flight mass spectrometry-based metabolome profiling of
WT/MF9 and Evi1/MF9 leukemia cells. ${ }^{23}$ We found significant differences between the cells in terms of the amounts of metabolites derived from the glycolytic and TCA cycles. The amounts of F1,6P and lactate in Evi1/MF9 leukemia cells were higher, implying activation of glycolysis. Moreover, the amounts of fumarate and malate (metabolites of the TCA cycle) were significantly higher in Evi1/MF9 cells (Figure 3A). To identify genes coexpressed with EVI1, we performed DNA microarray analysis of $\mathrm{GFP}^{+}$whole leukemia cells from WT/MF9 and Evi1/MF9 mice (Figure 3B and Online Supplementary Figure S2) (GSE118096), and compared the results with those for EVI1-over-expressing normal Lin ${ }^{\text {low }}$ BM cells (GSE34729). ${ }^{14}$

Expression profiling of Evi1-transduced normal BM cells ${ }^{14}$ and MF9 leukemia cells identified upregulation of Idh2 and downregulation of Idh1 in both (Figure 3B and Online Supplementary Figure S2); the expression of Idh1 and Idh2 was confirmed by quantitative real-time polymerase chain reaction (qRT-PCR) (Figure $3 \mathrm{C}$ ). Upregulation of Idh2 may induce increased production of metabolites such as fumarate and malate (metabolites of the TCA cycle). In addition, qRT-PCR revealed increased expression of genes involved in glycolysis including Glut-1 in whole AML cells (Figure 3C and Online Supplementary Figure S3). Collectively, these data indicate that EVI1 induces changes in glucose metabolism and the TCA cycle.

\section{EVI1 activates oxidative phosphorylation via glutaminolysis}

To determine how EVI1 regulates mitochondrial metabolism, we measured mitochondrial mass by flow cytometry after staining with MitoTracker Deep Red FM. We also measured mitochondrial superoxide production by staining cells with CellROX or MitoSOX. The mitochondrial mass in Evi1/MF9 cells was equivalent to that in WT/MF9 cells (Figure 4A); however, reactive ocygen species (ROS) levels and mitochondrial superoxide production were significantly higher in Evi1/MF9 cells (Figure 4B and C), suggesting that EVI1 expression may facilitate accumulation of ROS. Next, to determine which glycolysis and OXPHOS pathways are important for energy production in Evi1/MF9 cells, we examined the OCR/ECAR ratio in two MF9 murine leukemia lines and various human AML cell lines under conditions of basal respiration. Overall, a higher OCR/ECAR ratio was observed in Evi1/MF9 and EVI1 ${ }^{\text {high }}$ AML cell lines than in WT/MF9 and EVI1 ${ }^{\text {low }}$ AML cell lines, suggesting that energy production in EVI1 leukemia cells is mainly dependent on oxidative phosphorylation (Figure 4D and E). To measure the dependency, capacity, and flexibility of leukemia cells to oxidize three major mitochondrial fuels (glutamine, long-chain fatty acids and glucose), we used the XF Mito Fuel Flex Test to examine mitochondrial respiration in MF9 cells in the presence or absence of a specific inhibitor of each fuel pathway. Compared with those in WT/MF9 cells, mitochondria in Evi1/MF9 cells were more dependent on glutamine than on glucose and fatty acids (Figure 4F). Moreover, when Evil/MF9 or WT/MF9 cells were cultured in the presence of different low concentrations of glucose or glutamine, Evi1/MF9 cells survived significantly longer in the presence of $<0.1 \mathrm{~g} / \mathrm{L}$ glucose (Figure $4 \mathrm{G}$ ); however, survival decreased significantly when cultured in the presence of $<0.2 \mathrm{mM}$ glutamine (Figure $4 \mathrm{H}$ ), suggesting that survival of Evi1/MF9 cells is very dependent on the glutamine concentration. 
When Evi1/MF9 or WT/MF9 cells were treated with the glutaminase inhibitor BPTES, Evil/MF9 cells (but not WT/MF9 cells) were sensitive to inhibition of OCR (Figure 4I). Collectively, these data indicate that EVI1 plays an essential role in mitochondrial energy reprogramming in MF9 AML cells by activating glutaminolysis (Figure 4J).

To identify the specific mutation that drives this metabolic phenotype (i.e. increased OXPHOS capacity in Evi1/MF9 cells), we measured OXPHOS levels and performed glucose/glutamine starvation experiments on cells infected with Evi1-targeting shRNA (shEvi1) or cells treated with a DOT1L inhibitor (EPZ004777). We found that
shEvi1 and EPZ004777 suppressed OXPHOS in Evi1/MF9 cells (Online Supplementary Figure S4A). When cells transfected with shEvi1 or treated with EPZ004777 were cultured in the presence of different low concentrations of glucose or glutamine, shEvil cells survived for a significantly longer time in lower concentrations of glutamine (Online Supplementary Figure S4B). This suggests that EVI1 is important for glutamine dependency. We performed quantitative PCR analysis of Glut-1, Idh1, and Idh2, and found that expression of Idh2 was lower in shEvi1 and in Evi1/MF9 cells treated with EPZ004777 (Online Supplementary Figure S4C). To investigate whether altered
A

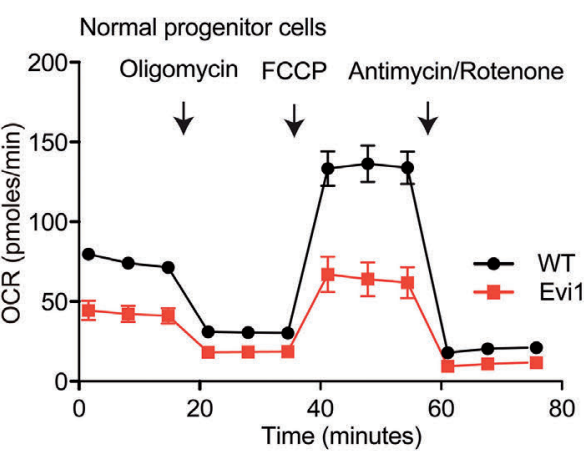

C

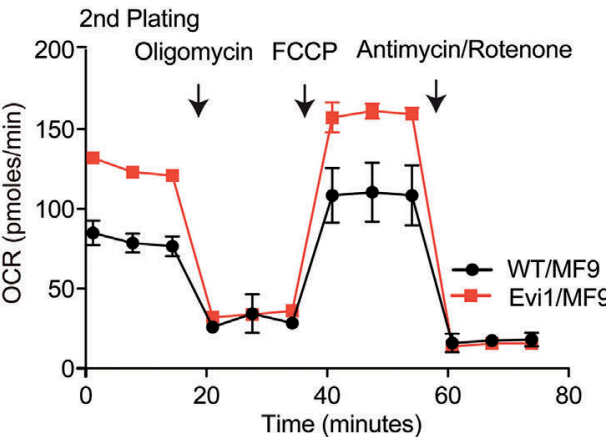

E

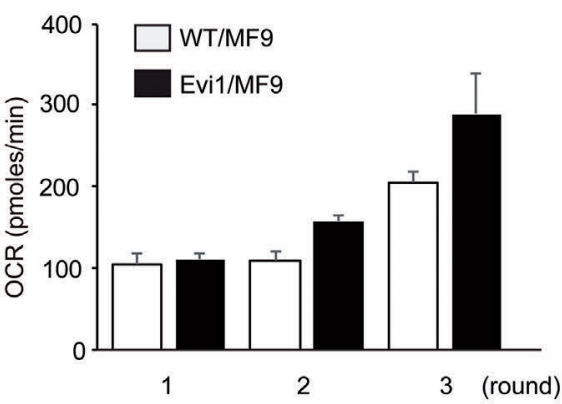

G

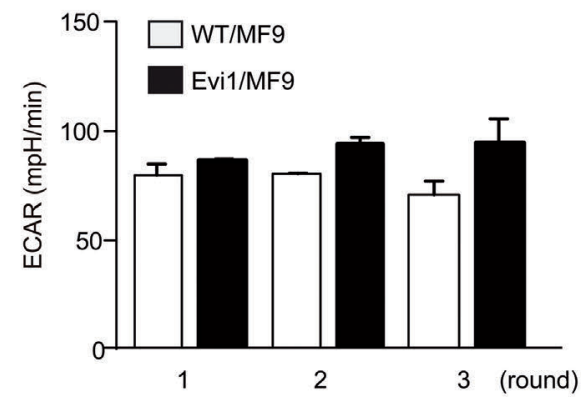

B

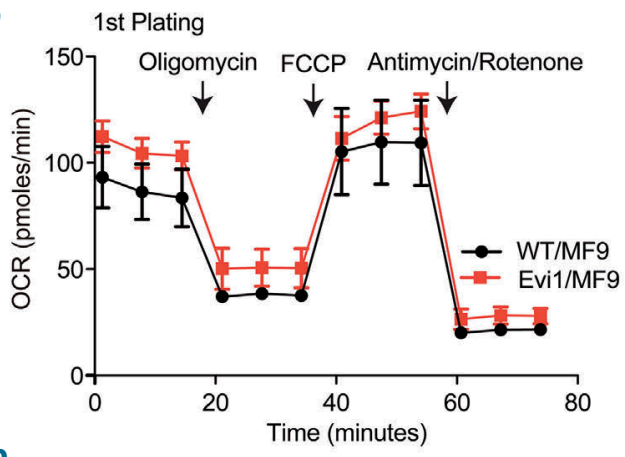

D

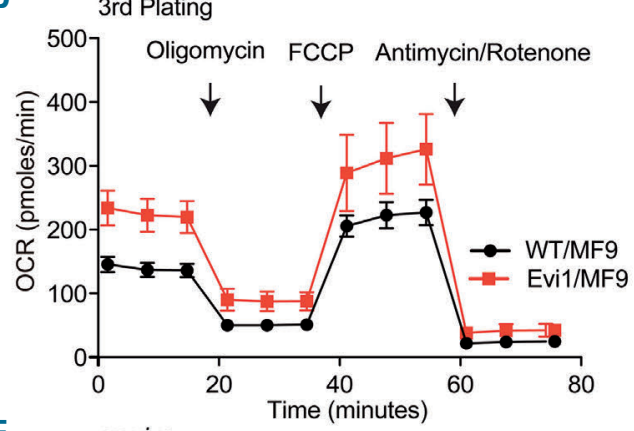

F

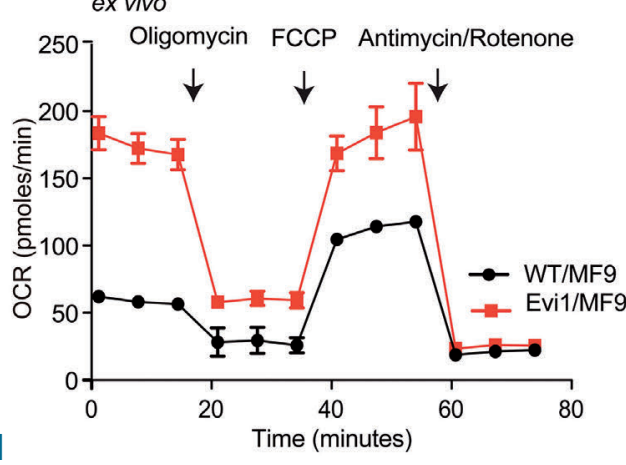

H

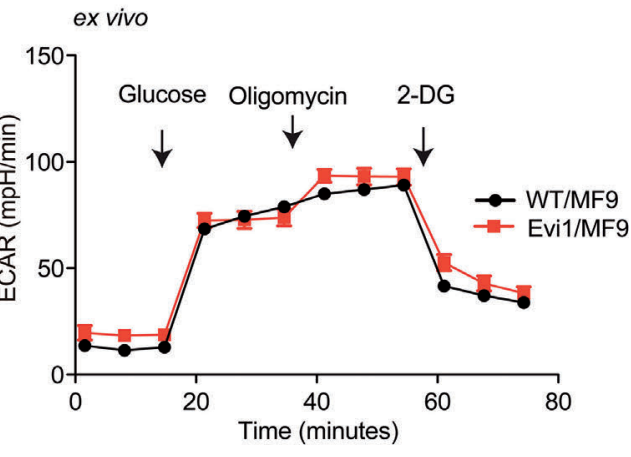

Figure 2. EVI1 increases oxidative phosphorylation (OXPHOS) and glycolysis during development of MLL-AF9 leukemia. (A) The basal oxygen consumption rate (OCR) and maximal respiratory capacity in Evi1-TG normal progenitor cells were significantly lower than those in wild-type (WT) progenitor cells. (B-D) After the $2^{\text {nd }}$ plating, the OCR of Evi1-TG progenitor cells infected with MLL-AF9 increased gradually when compared with that of WT cells. (E) After the $2^{\text {nd }}$ plating, the maximal respiratory capacity of Evi1/MF9 increased gradually when compared with that of WT cells. (F) The basal and capacity OCR of EVI1/MF9 bone marrow cells were significantly higher than those of WT/MF9 cells. (G) The basal ECAR of Evi1/MF9 cells increased slightly after the $3^{\text {tr }}$ plating. $(\mathrm{H})$ There was no significant difference between ECAR of ex vivo leukemia cells from WT/MF9 and from Evi1/MF9 mice. All samples were collected at least in duplicate. 


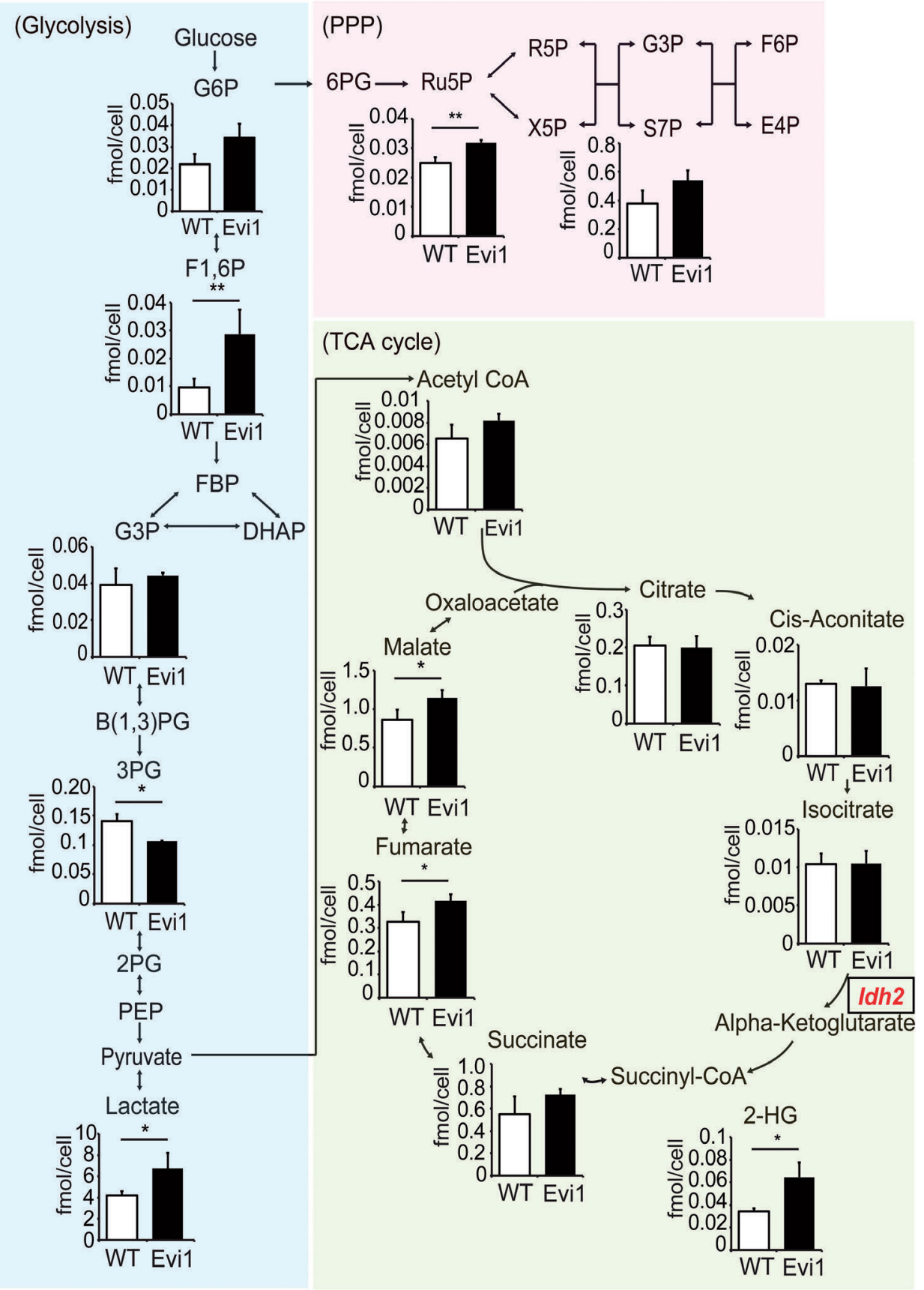

C

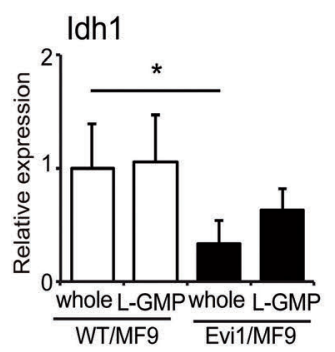

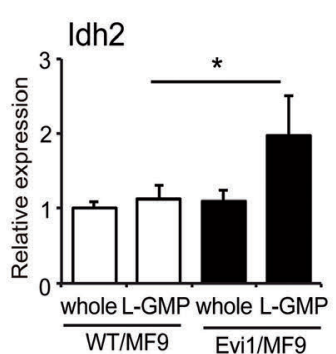

Glut-1

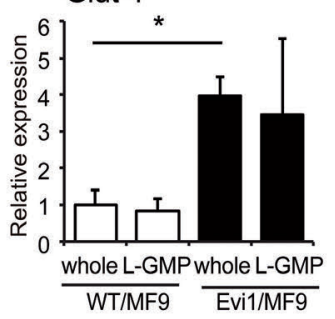

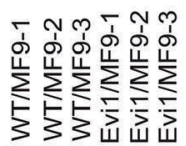
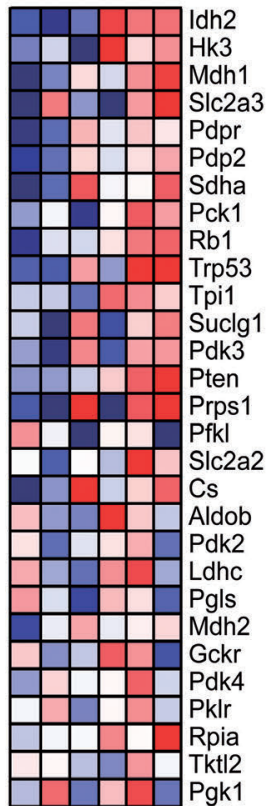

Ckmt1

Gpd2
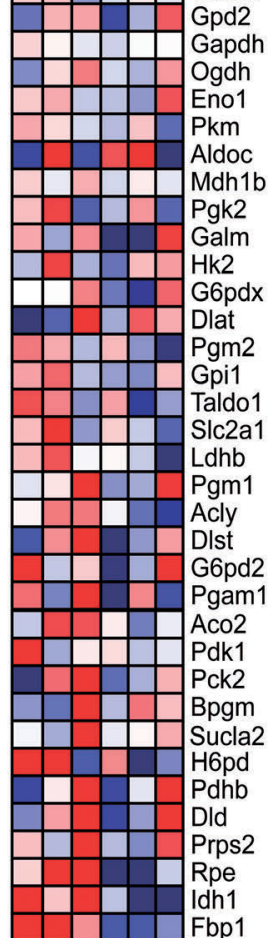

Relative

Row min

Figure 3. Expression of EVI1 is associated with metabolic reprograming. (A) "Map" of the metabolic pathways and metabolite concentrations in the glycolysis, PPP, and TCA cycle pathways. The concentrations of F1,6P, lactate, fumarate, and malate were significantly higher in Evi1/MF9 cells ( $\mathrm{n}=3$ ). (B) Heat map of genes encoding metabolism-related factors differentially expressed upon genomic profiling of WT/MF9 or Evi1/MF9 leukemia cells ( $n=3$ ). (C) Expression of Idh1, Idh2, and Glut1 operating in the glycolytic pathways and TCA cycle, as measured by qRT-PCR. Expression was normalized to that of $\beta$-actin and to that of whole WT/MF9 cells ( $\mathrm{n}=4$ ). 
A
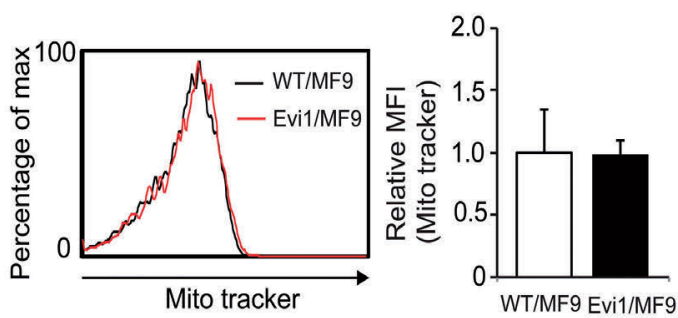

C

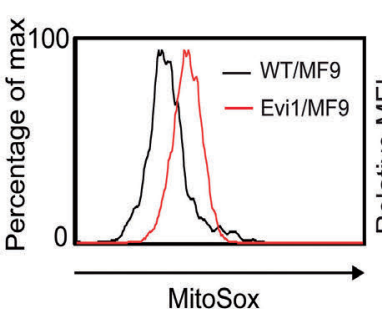

E

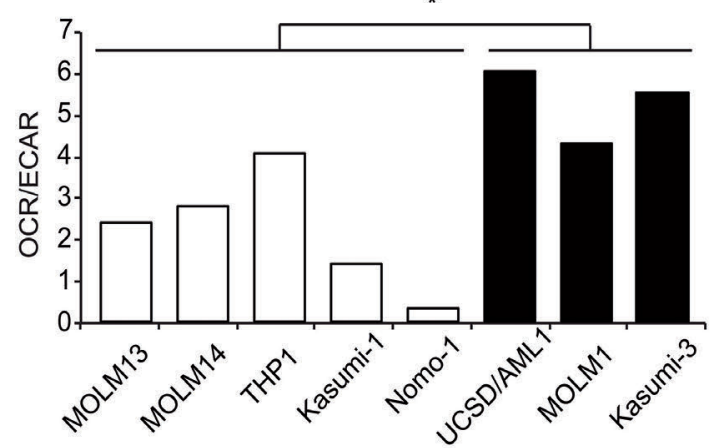

G

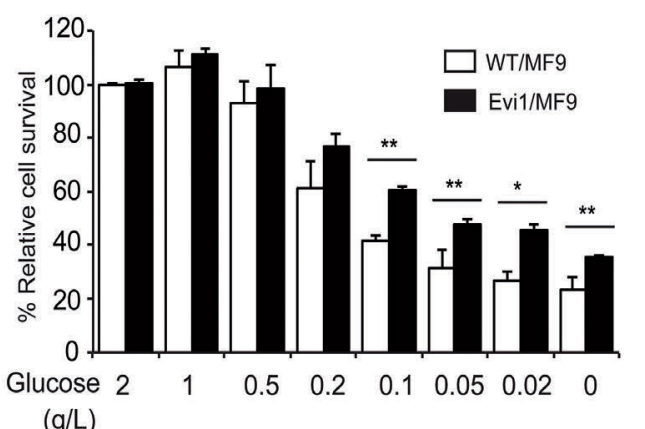

B

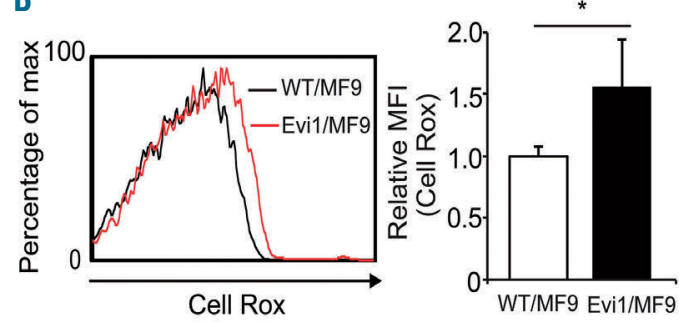

D

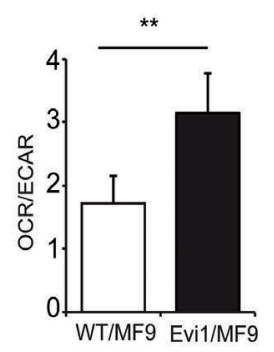

$F$

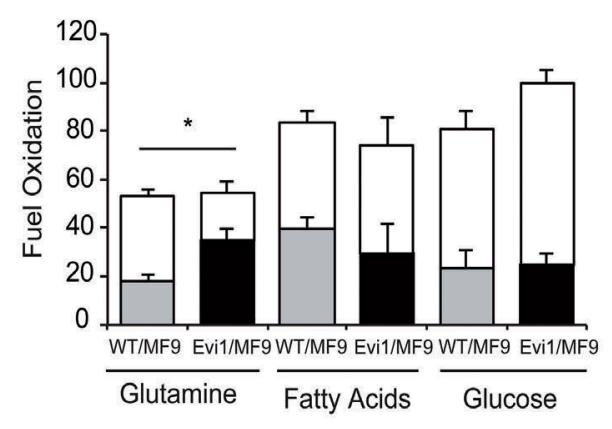

$\mathrm{H}$

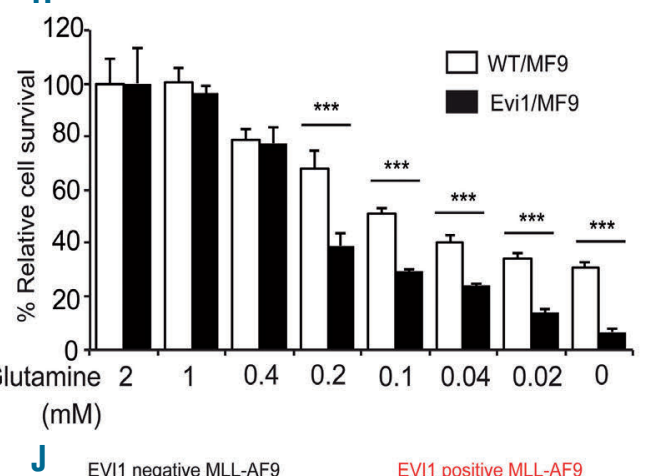

J EVI1 negative MLL-AF9 EVI1 positive MLL-AF9

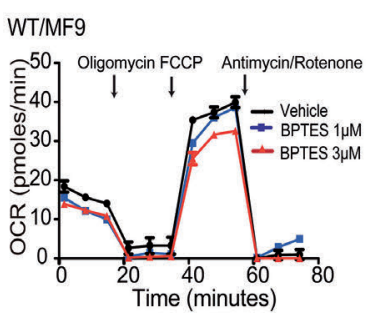

Evi1/MF9

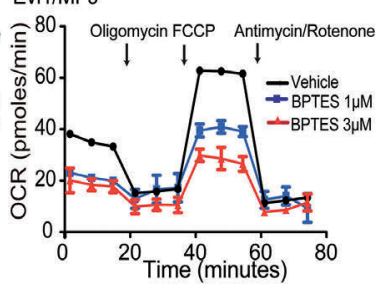

Glucose

Glucose

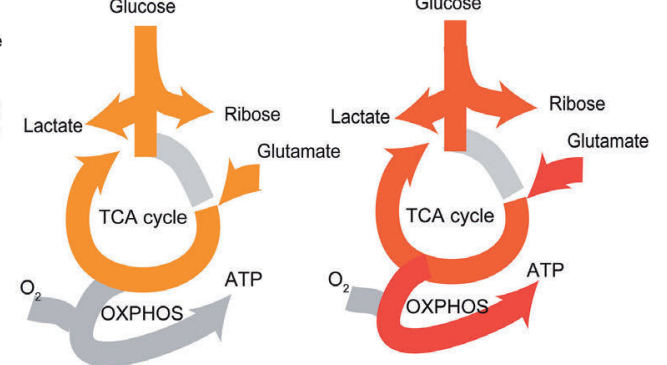

Figure 4. EVI1 activates oxidative phosphorylation (OXPHOS) via glutaminolysis. (A) Mitochondrial mass in Evi1/MF9 (red line) cells was equivalent to that in WT/MF9 (black line) ( $n=3$ ). (B and C) Reactive oxygen species (ROS) levels and mitochondrial superoxide production were significantly higher in Evi1/MF9 ( $n=3$ ). ( and E) The oxygen consumption rate (OCR)/extracellular acidification rate (ECAR) ratio under conditions of basal respiration in Evi1/MF9 (D) and EVI ${ }^{\text {high }}$ acute myeloid leukemia (AML) cell lines (E) was significantly higher than that in WT/MF9 cells and EVI1 $1^{\text {low }}$ AML cell lines. (F) The XF Mito Fuel Flex Test calculates the rate of oxidation of a given fuel as a percentage of oxidation of all three fuels being tested. Fuel dependency (black or gray) is a measurement of reliance on a particular fuel pathway to maintain baseline respiration. Fuel flexibility (white) is the ability of cells to increase oxidation of a particular fuel to compensate for inhibition of an alternative fuel pathway. Evi1/MF9 showed an increased dependency on glutamine, but not glycolysis and fatty acids. (G) Evi1/MF9 cells showed increased resistance to glucose starvation. (H) Evi1/MF9 cells were sensitized to glutamine starvation. ${ }^{*} P<0.05 ; * * P<0.005$; $* * * P<0.0005$ (Student's $t$-test). (I) The OCR of glutaminolysis inhibitor (BPTES)-treated WT/MF9 leukemia cells remained unchanged. The basal and maximum OCR of BPTES-treated Evi1/MF9 leukemia cells were lower than those of vehicle-treated cells. $(\mathrm{J})$ Schematic representation of metabolic reprogramming upon EVI1 expression. 
expression of Idh1/Idh2 is functionally linked to the increased OXPHOS levels observed in Evi1/MF9 cells, we measured OXPHOS in Evi1/MF9 cells transfected with shIdh1 or shIdh2. Evi1/MF9-shIdh2 cells showed markedly lower levels of OXPHOS than shScr- and shIdh1-transfected cells (Online Supplementary Figure S4D). This suggests that upregulation of Idh2 via EVI1 may increase OXPHOS activity, which is important for energy production in Evi1/MF9 cells.

\section{L-asp inhibits EVI1-mediated oxidative phosphorylation activity}

To develop a novel strategy for treatment of Evi1/MF9 cells, we tested the effect of specific inhibitors of glycolysis by 2-deoxyglucose (2-DG) and STF31 (a GLUT-1 inhibitor) and glutaminolysis by BPTES (a glutamine synthetase inhibitor); L-asparaginase (L-asp) is an enzyme that catalyzes hydrolysis of asparagine and glutamine.

Evil/MF9 cells were more sensitive to glycolysis and glutaminolysis inhibitors than WT leukemia cells (Figure $5 \mathrm{~A})$. Moreover, treatment with L-asp led to marked suppression of Evi1/MF9 cell growth (Figure 5B). Analysis of several human AML cells revealed that the $\mathrm{IC}_{50}$ of $\mathrm{L}$-asp against three EVI1 high AML cell lines and two primary EVI1 $^{+}$AML cells was lower than that against EVI1 low AML cell lines and two primary EVI1- AML cells (Figure 5C and D). To confirm whether L-asp treatment affects mitochondrial respiration, we used the XFp extracellular flux analyzer to measure energy metabolism by murine Evi1/MF9 and human EVI1 ${ }^{+} \mathrm{AML}$ cells after L-asp treatment. The basal and maximum OCR values for mitochondria in L-asp-treated Evi1/MF9 cells and human EVI1+AML cells were markedly lower than in non-treated cells, suggesting that EVI1 ${ }^{+}$AML cells are more sensitive to L-asp (Figure 5E). WT/MF9 and Evi1/MF9 do not express surface markers for B- and T-lymphoid cells (Online Supplementary Figure S5A). Addition of L-asp led to a marked reduction in the OCR of normal progenitor cells in Evi1-TG mice (Online Supplementary Figure S5B); however, there was no difference in the $\mathrm{IC}_{50}$ between WT and Evi1-TG mice (Online Supplementary Figure S5C). Some metabolic pathways may play a role in normal BM progenitor cells but not in leukemia cells. To examine whether suppression of OCR by L-asp depends on glutamine depletion, we studied the effects of high glutamine concentration on the OCR of cultured cells. In WT/MF9, L-asp treatment showed no OCR-suppressing effect, which was not affected by glutamine concentration. Meanwhile, in Evi1/MF9, high concentration of glutamine attenuated the OCR-suppressing effect (Online Supplementary Figure S5D). Taken together, these results indicate that L-asp treatment suppresses growth of EVI1+ AML cells by blocking EVI1mediated OXPHOS activity.

To examine the therapeutic potential of L-asp in vivo, recipient mice transplanted secondarily with Evi1/MF9 AML cells were treated with L-asp via intraperitoneal injection beginning on day 5 post transplantation; L-asp $(1,000 \mathrm{U} / \mathrm{kg})$ was injected five times per week for four weeks (Figure 6A). L-asp treatment led to a significant reduction in the number of $\mathrm{GFP}^{+} \mathrm{AML}$ cells in the peripheral blood (Figure $6 \mathrm{~B}$ ) and extended the survival time of recipient mice (Figure $6 \mathrm{C}$ ). Although L-asp treatment reduced the total number of leukemia cells in the BM, there was no increase in the number of L-GMP cells in the BM (Figure 6D). Moreover, the basal and maximum OCR in L-asp-treated BM cells were significantly lower than those in untreated cells (Figure 6E). By contrast, L-asp treatment did not reduce the number of leukemia cells in WT/MF9 mice and did not prolong their survival (Online Supplementary Figure S6A-C). Next, we examined the effect of L-asp treatment in a subcutaneous xenograft model of human AML. Immunodeficient NOG mice were injected with UCSD/AML1 cells and treated with L-asp (Figure 6F). L-asp treatment suppressed the growth of human EVI1 ${ }^{\text {high }}$ AML tumors effectively (Figure 6G-I). Overall, these findings indicate that inhibition of OXPHOS by L-asp is a potential effective treatment for EVI1 ${ }^{\text {high }}$ AML.

\section{Low expression of ASNS by MLL-AF9 leukemia cells is associated with high sensitivity to L-asp}

High sensitivity to L-asp is due to active glutaminolysis and low expression of asparagine synthetase (Asns). Therefore, we examined expression of the glutamine transporter (Slc1a5) and Asns in MF9 cells. Expression of Asns by Evi1/MF9 cells was significantly lower than that by WT/MF9 cells (Figure 7A). By contrast, expression of Slc1a5 by Evi1/MF9 cells was significantly higher than that by WT/MF9 cells (Figure 7B). WT/MF9 cells transfected with shAsns showed a lower IC50 for L-asp (Online Supplementary Figure S7). These findings suggest that sensitivity to L-asp correlates with ASNS expression in MF9 leukemia.

Next, we examined expression of IDH2, ASNS, and SLC1A5 in human leukemia cell lines. L-asp-sensitive EVI1 $^{\text {high }}$ AML cell lines and two primary EVI1 ${ }^{+}$AML cells showed low expression of ASNS (Online Supplementary Figure S8A and B).

Finally, to determine whether these genes are clinically useful markers for predicting L-asp sensitivity, we examined their expression in leukemia samples from patients in JPLSG AML-05. In the MLL-r AML cohort ( $n=48$ ), there was no significant difference in ASNS expression between EVI1 $^{-}$and EVI1 ${ }^{+}$AML (Figure 7C). However, expression of ASNS in MF9 AML was significantly lower than that in non-MF9 AML (Figure 7D). Moreover, expression of ASNS in EVI1 ${ }^{+}$MF9 AML ( $\left.=11\right)$ was significantly lower than that in those with EVI1-MF9 ( $n=17)$; however, EVI1 expression did not affect ASNS expression in non-MF9 AML ( $n=20)$ (Figure 7E and F). We found no significant difference between $\mathrm{EVI1}^{+}$and EVI1- MF9 AML patients with respect to expression of IDH2 and SLC1A5 (Figure 7G and $\mathrm{H})$. These findings suggest that lower expression of ASNS in EVI1 $1^{+} \mathrm{MF9}$ AML is associated with higher sensitivity to L-asp.

\section{Discussion}

Previously, it was thought that activation of glycolysis was responsible primarily for metabolic reprogramming at onset of leukemia. In addition, clones that promote the survival of BM cells are produced when genes involved in onset of leukemia directly activate glycolytic metabolism. ${ }^{24-27}$ However, recent studies report a direct connection between mitochondrial metabolic activity and refractory AML; this is because: (i) production of adenosine triphosphate (ATP) by mitochondria is higher in leukemia stem cells than in differentiated blast cells or normal HSC; (ii) chemotherapy-resistance of AML correlates 
A
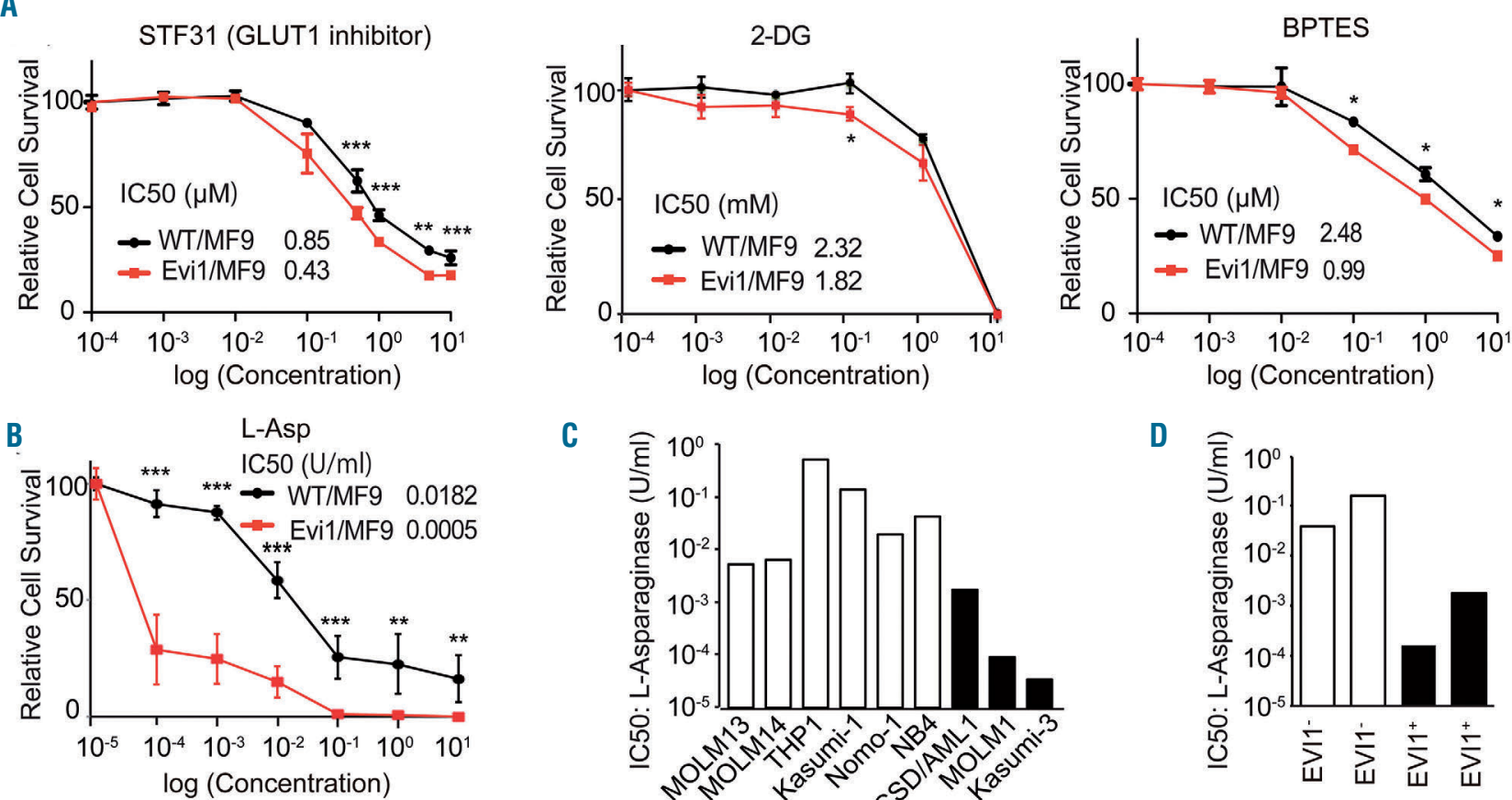

C

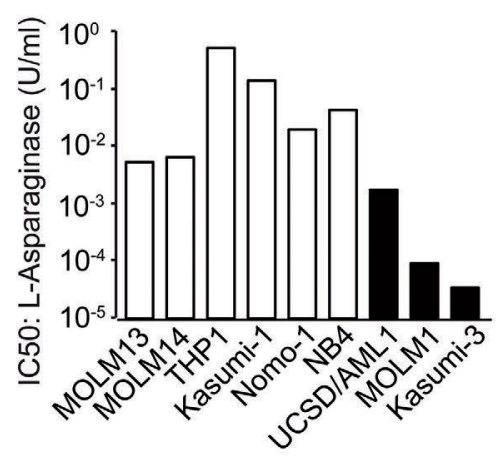

Evi1/MF9

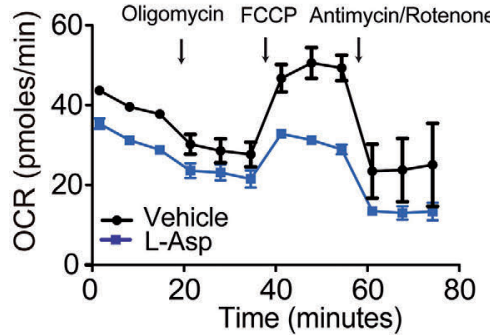

NB4

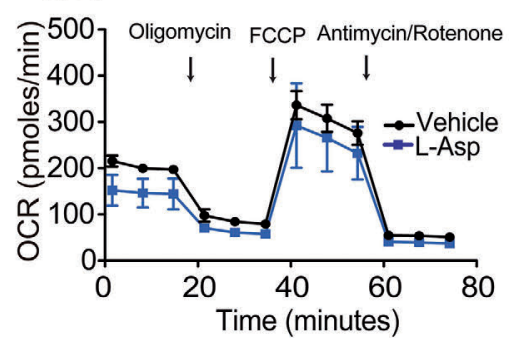

EVI1-

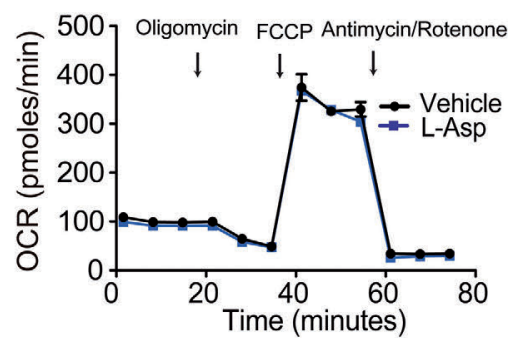

D

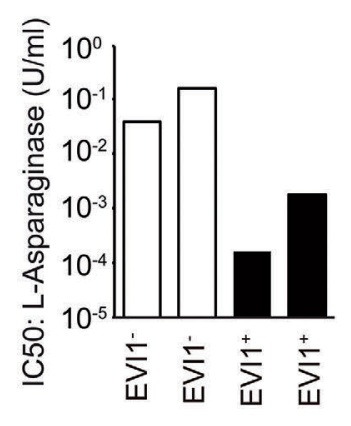

WT/MF9

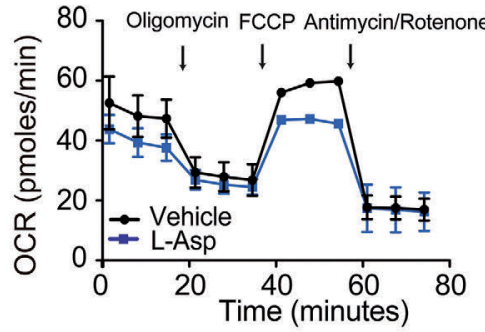

Kasumi-1

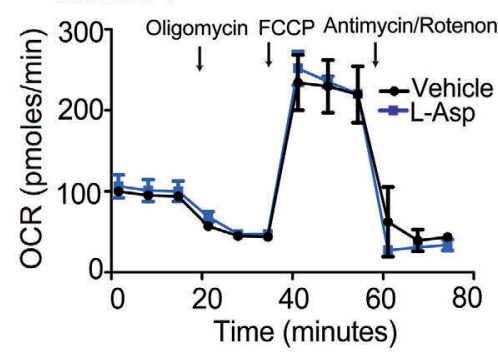

MOLM1

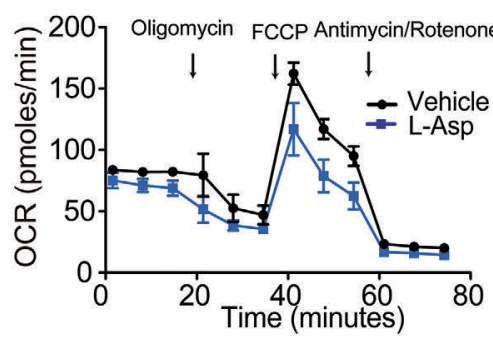

THP1

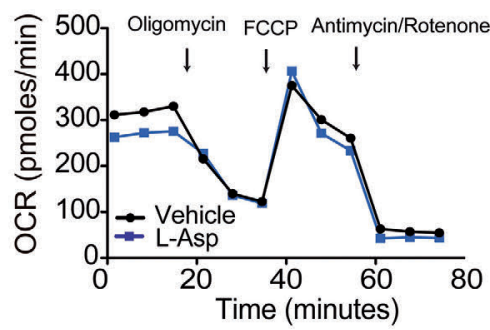

UCSD/AML1

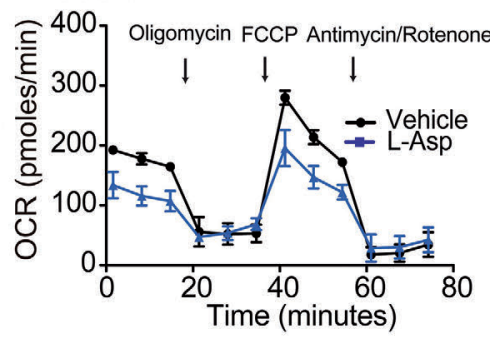

EVI1 ${ }^{+}$

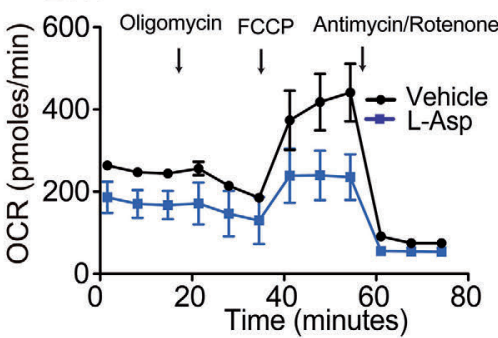

Figure 5. L-asparaginase (L-asp) inhibits EVI1-mediated activation of oxidative phosphorylation (OXPHOS). (A) Survival of MF9 acute myeloid leukemia (AML) cells after brief exposure to glycolysis inhibitors (STF31 and 2-DG) and a glutaminolysis inhibitor (BPTES). Evi1/MF9 cells were more sensitive to glycolysis and glutaminolysis inhibitors than wild-type (WT) leukemia cells. (B) L-asp suppressed proliferation of Evi1/MF9 cells. (C) EVI $1^{\text {high }} A M L$ cell lines were more sensitive to L-asp than EVI1 ${ }^{\text {low }}$ AML cell lines. (D) AML cells from two EVI1 ${ }^{+}$AML patients were more sensitive to L-asp than those from EVI1- AML patients. (E) Energy metabolism was analyzed by the XFp extracellular flux analyzer after L-asp treatment $(1 \mathrm{U} / \mathrm{mL})$ of $A M L$ cells. Basal and maximum OCR in mitochondria were much lower in L-asp-treated Evi1/MF9 than in L-asp-treated WT/MF9 cells. L-asp does not suppress mitochondrial oxidation in EVI1 ${ }^{\text {low }}$ AML cell lines (THP1, Kasumi-1 and NB4) and primary EVI1 AML cells. L-asp suppressed basal and maximum OCR in mitochondria of EVI1 1igh AML cell lines (UCSD/ANL1, MOLM1) and primary EVI1 ${ }^{+}$leukemia cells. 
strongly with OXPHOS activation to a greater extent than with leukemic stemness; and (iii) high OXPHOS activity in cells showing stemness properties induces tyrosine kinase inhibitor-resistance in those with chronic myelocytic leukemia. ${ }^{21,22,28}$ Here, we found that OXPHOS is activated before glycolysis during onset of leukemia, thereby reconfirming the importance of mitochondrial metabo- lism. A previous study reports that EVI1 plays an important role in metabolic regulation of leukemia, and that a metabolic pathway involving creatinine is the key. ${ }^{14}$ However, it is unclear how EVI1 controls metabolism and contributes to malignant alterations in MLL-r AML cells. The leukemia mouse model used in the present study did not show any alterations in creatine gene expression;
A

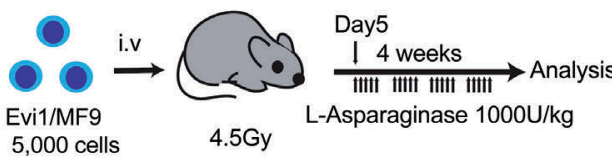

D

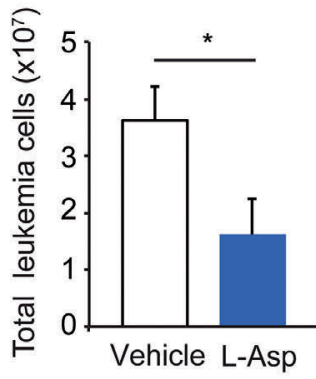

$\mathbf{F}$

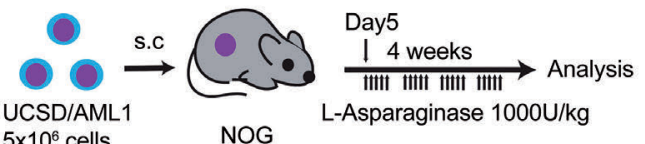

H

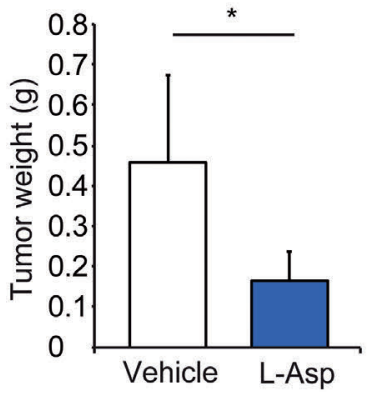

I
B

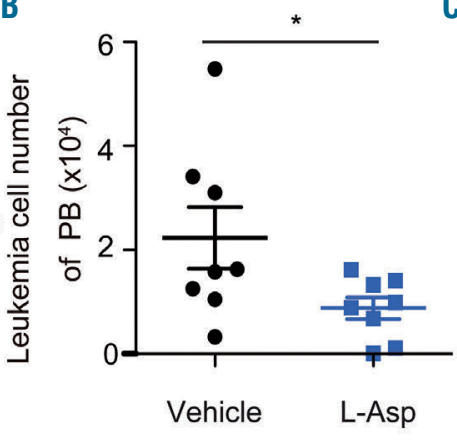

C

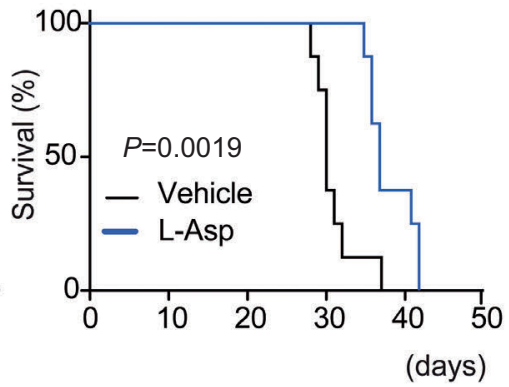

$\mathrm{E}$
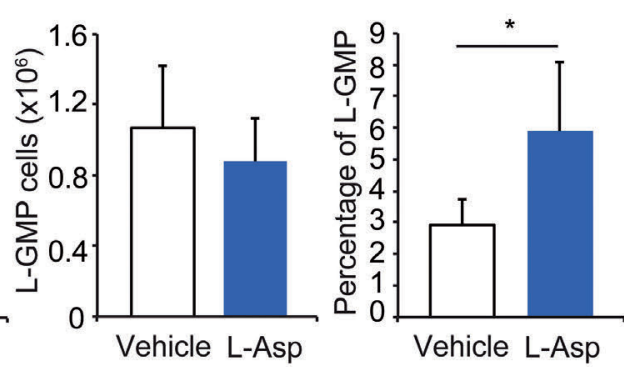

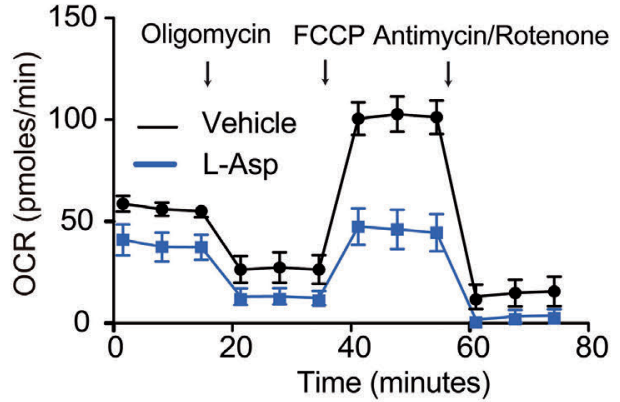

G
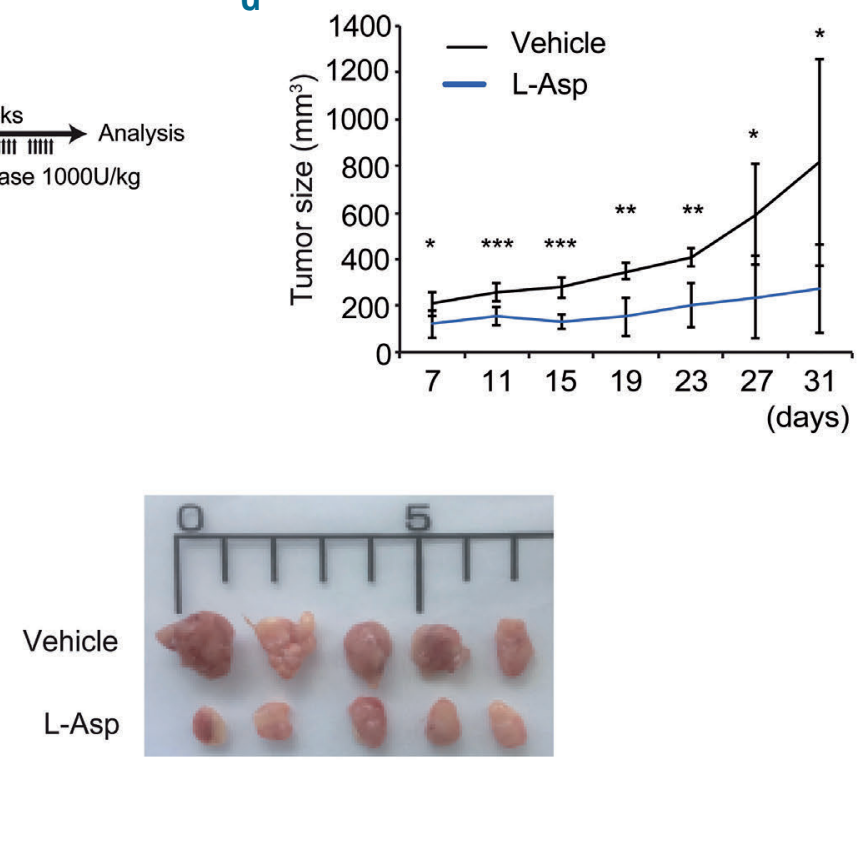

Figure 6. L-Asp inhibits growth of EVI1 ${ }^{\text {high }}$ acute myeloid leukemia (AML) tumor cells in vivo. (A) Schematic outline of the Evi1/MF9 mouse model treated with L-asp. Treatment began five days post transplantation. (B) L-asp led to a significant reduction in the number of circulating $\mathrm{GFP}^{+} \mathrm{AML}$ cells in peripheral blood ( $\mathrm{n}=8$ ). (C) L-asp treatment extended the survival of recipient mice significantly $(n=8)$. (D) The total number of whole AML cells in the bone marrow fell significantly after exposure to L-asp, but the percentage of L-GMP increased significantly. (E) The oxygen consumption rate (OCR) of L-asp-treated leukemia cells in MF9 mice was lower than that of vehicle-treated cells. (F) Schematic outline of the UCSD/AML1 xenograft mouse model treated with L-asp. Treatment began five days post transplantation. (G-I) L-asp inhibited growth of AML tumors significantly $(G)$. Tumor weight $(H)$ and tumor size $(I)(n=5)$. 
however, it did show that an advantage in terms of mitochondrial metabolism-dependent energy production could be obtained by activating glutamine metabolism. Furthermore, we suggest that high EVI1 expression maintains OXPHOS activity, even in differentiated cells, and contributes to treatment resistance. Although we did not identify the genes controlled directly by EVI1, we believe that ASNS, IDH2, and SLC1A5 are candidates. IDH2 is mutated in $8-19 \%$ of AML cases; therefore, a mutant
IDH2 inhibitor is under development. Future studies should examine whether metabolic reprogramming or increased 2-HG production are induced upon high expression of IDH2. ${ }^{29}$ In addition, we identified L-asp as a metabolic inhibitor for EVI1 ${ }^{+}$leukemia. L-asp exerts an antitumor effect by depleting both asparagine and glutamine. ${ }^{30,31}$ Sensitivity to L-asp correlates with ASNS expression, but the clinical marker is unknown. ${ }^{32}$

Although some AML cases are highly sensitive to L-asp,
A

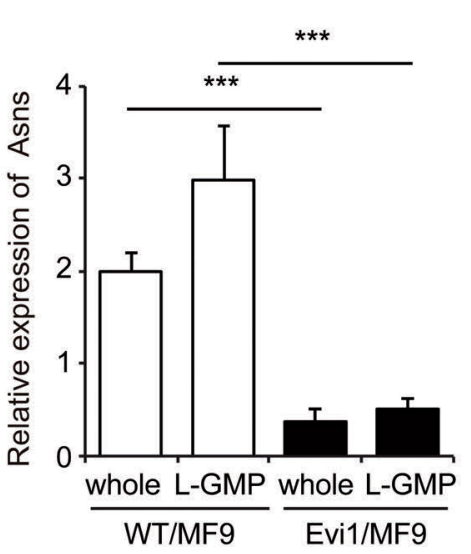

D

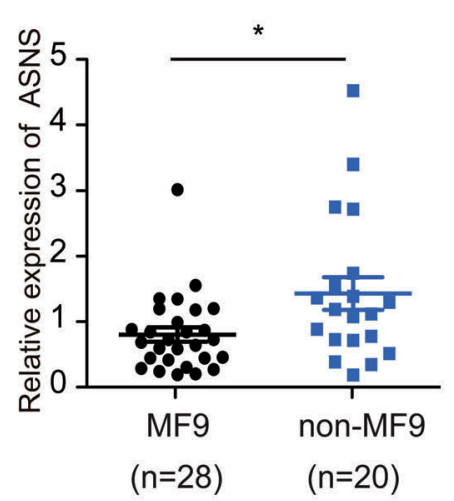

G

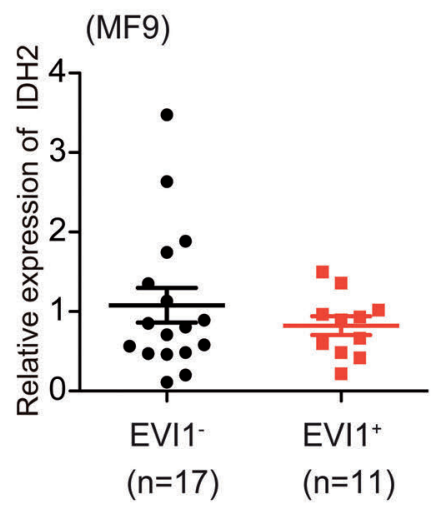

B

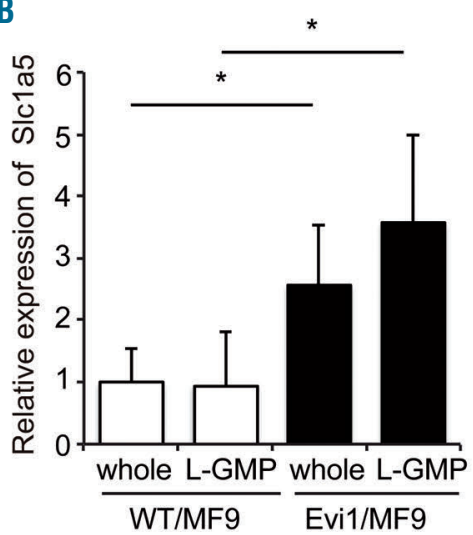

E

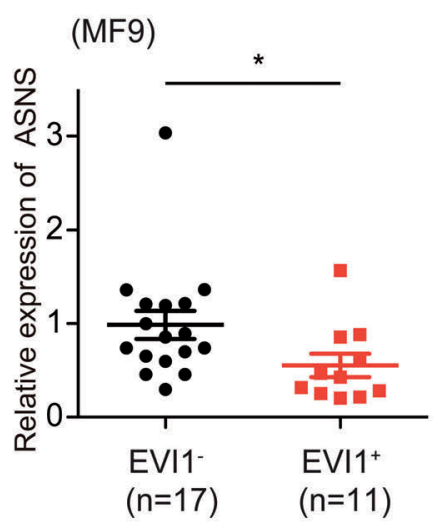

C

(All)

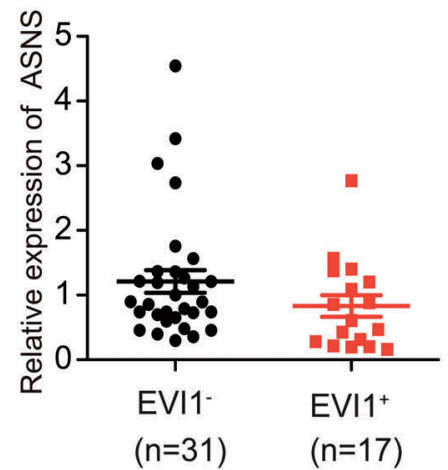

F

(non-MF9)

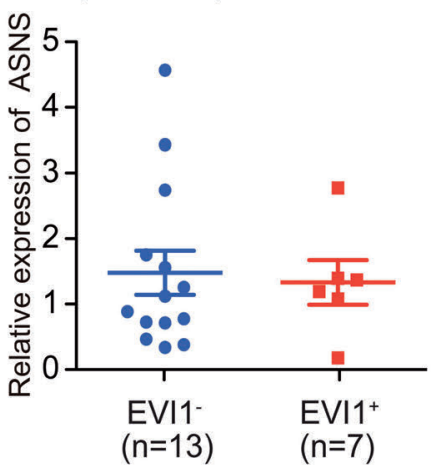

H

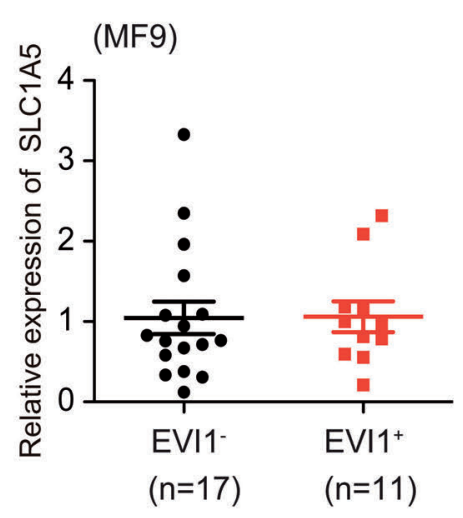

Figure 7. Low expression of ASNS in MF9 leukemia cells increases their sensitivity to L-asp. (A and B) Expression of the glutamine transporter (SIc1a5) and ASNS by MF9 leukemia cells. Expression of ASNS by Evi1/MF9 cells was significantly lower than that by wild-type (WT) cells. Expression of SIc1a5 by Evi1/MF9 cells was significantly higher than that by WT cells. (C-H) Gene expression in samples from JPLSG AML-05. In the acute myeloid leukemia (AML) with MLL rearrangement (MLL$r$ AML) cohort $(n=48)$, there was no significant difference in ASNS expression between those with EVI1- and those with EVI $1^{+}$AML $(C)$. (D) MF9 AML showed significantly lower expression of ASNS than non-MF9 MLL-r AML. Expression of ASNS by EVI1- MF9 cells was significantly lower than that of EVI1- MF9 ( $n=28$ ) (E) but not that of the non-MF9 cohort $(n=20)(F)$. $(G$ and H) Expression of IDH2 $(G)$ and SLC1A5 $(H)$ in the MF9 cohort. 
the underlying mechanism is unclear. ${ }^{33} \mathrm{AML}$ showing high EVI1 expression is often accompanied by monosomy 7. Reduced expression of ASNS in AML with monosomy 7 is caused by haplodeletion of chromosome 7, making it highly sensitive to L-asp. ${ }^{34}$ Here, since EVI1 ${ }^{+}$AML showed high sensitivity to L-asp despite its low glutamine dependence, it is likely that monosomy 7 is involved. Furthermore, we clarified that increased glutamine dependency by MF9 AML cells showing high EVI1 expression and low ASNS expression makes them sensitive to Lasp. Blockade of the creatine kinase pathway, which is essential for mitochondrial respiration, reduces glutamate levels in EVI1 ${ }^{+}$AML cells. ${ }^{14}$ Future studies should examine whether expression of ASNS by refractory MLL-r AML may increase the therapeutic potential of L-asp and improve treatment outcomes.
In conclusion, we found that the energy advantage of AML cells is acquired via transcription factor-mediated activation of mitochondrial metabolism, leading to a poor prognosis. Furthermore, we show that new therapeutic options can be identified by examining the energy-based metabolic characteristics of leukemia cells.

\section{Funding}

This research was supported by JSPS KAKENHI Grants (Number JP.16K19581 and AMED under Grant Number JP. $17 \mathrm{~cm} 0106126 h 0002)$ and grants from the Takeda Science Foundation, the Friends of Leukemia Research Fund, The Shinnihon Foundation of Advanced Medical Treatment Research, and the Japanese Society of Hematology Research. Microarray data have been deposited in the Gene Expression Omnibus (GSE118096). The authors have no conflicts of interest to declare.

\section{References}

1. Muntean AG, Hess JL. The pathogenesis of mixed-lineage leukemia. Annu Rev Pathol. 2012;7:283-301.

2. Balgobind BV, Zwaan CM, Pieters R, Van den Heuvel-Eibrink MM. The heterogeneity of pediatric MLL-rearranged acute myeloid leukemia. Leukemia. 2011;25(8): 1239-1248.

3. Morishita K, Parker DS, Mucenski ML, et al. Retroviral activation of a novel gene encoding a zinc finger protein in IL-3-dependent myeloid leukemia cell lines. Cell. 1988; 54(6):831-840.

4. Morishita K, Parganas E, William CL, et al. Activation of EVI1 gene expression in human acute myelogenous leukemias by translocations spanning 300-400 kilobases on chromosome band 3q26. Proc Natl Acad Sci U S A. 1992;89(9):3937-3941.

5. Lugthart S, Gröschel S, Beverloo HB, et al. Clinical, molecular, and prognostic significance of WHO type inv(3)(q21q26.2)/t(3;3) (q21; $q 26.2$ ) and various other $3 \mathrm{q}$ abnormalities in acute myeloid leukemia. J Clin Oncol. 2010;28(24):3890-3898.

6. Gröschel S, Schlenk RF, Engelmann J, et al. Deregulated expression of EVI1 defines a poor prognostic subset of MLL-rearranged acute myeloid leukemias: a study of the German-Austrian Acute Myeloid Leukemia Study Group and the Dutch-Belgian-Swiss HOVON/SAKK Cooperative Group. J Clin Oncol. 2013;31(1):95-103.

7. Matsuo H, Kajihara M, Tomizawa D, et al. EVI1 overexpression is a poor prognostic factor in pediatric patients with mixed lineage leukemia-AF9 rearranged acute myeloid leukemia. Haematologica. 2014;99(11):e225227.

8. Valk PJ, Verhaak RG, Beijen MA, et al. Prognostically useful gene-expression profiles in acute myeloid leukemia. N Engl J Med. 2004;350(16):1617-1628.

9. Verhaak RG, Wouters BJ, Erpelinck CA, et al. Prediction of molecular subtypes in acute myeloid leukemia based on gene expression profiling. Haematologica. 2009;94(1):131134.

10. Saito Y, Nakahata S, Yamakawa N, et al. CD52 as a molecular target for immunotherapy to treat acute myeloid leukemia with high EVI1 expression. Leukemia. 2011; 25(6):921-931.
11. Saito Y, Kaneda K, Suekane A, et al Maintenance of the hematopoietic stem cell pool in bone marrow niches by EVI1-regulated GPR56. Leukemia. 2013;27(8):1637 1649 .

12. Wise DR, DeBerardinis RJ, Mancuso A, et al. Myc regulates a transcriptional program that stimulates mitochondrial glutaminolysis and leads to glutamine addiction. Proc Natl Acad Sci U S A. 2008;105(48):1878218787

13. Cantor JR, Sabatini DM. Cancer cell metabolism: one hallmark, many faces. Cancer Discov. 2012;2(10):881-898.

14. Fenouille N, Bassil CF, Ben-Sahra I, et al. The creatine kinase pathway is a metabolic vulnerability in EVI1-positive acute myeloid leukemia. Nat Med. 2017;23(3):301-313.

15. Zhang Y, Owens K, Hatem L, et al. Essential role of PR-domain protein MDS1-EVI1 in MLL-AF9 leukemia. Blood. 2013;122(16): 2888-2892.

16. Stavropoulou V, Kaspar S, Brault L, et al. MLL-AF9 Expression in Hematopoietic Stem Cells Drives a Highly Invasive AML Expressing EMT-Related Genes Linked to Poor Outcome. Cancer Cell. 2016;30 (1):43 58

17. Ng CE, Yokomizo T, Yamashita N, et al. A Runx1 intronic enhancer marks hemogenic endothelial cells and hematopoietic stem cells. Stem Cells. 2010;28(10):1869-1881.

18. Saito Y, Chapple RH, Lin A, Kitano A Nakada D. AMPK Protects LeukemiaInitiating Cells in Myeloid Leukemias from Metabolic Stress in the Bone Marrow. Cell Stem Cell. 2015;17(5):585-596.

19. Krivtsov AV, Armstrong SA. MLL translocations, histone modifications and leukaemia stem-cell development. Nat Rev Cancer. 2007;7(11):823-833

20. Somervaille TC, Cleary ML. Identification and characterization of leukemia stem cells in murine MLL-AF9 acute myeloid leukemia. Cancer Cell. 2006;10(4):257-268.

21. Farge T, Saland E, de Toni F, et al. Chemotherapy-resistant human acute myeloid Leukemia cells are not enriched for leukemic stem cells but require oxidative metabolism. Cancer Discov. 2017;7(7):716735.

22. Kuntz EM, Baquero P, Michie AM, et al. Targeting mitochondrial oxidative phosphorylation eradicates therapy-resistant chronic myeloid leukemia stem cells. Nat Med. 2017;23(10):1234-1240.
23. Hirayama A, Kami K, Sugimoto M, et al Quantitative metabolome profiling of colon and stomach cancer microenvironment by capillary electrophoresis time-of-flight mass spectrometry. Cancer Res. 2009;69(11): 4918-4925.

24. Vander Heiden MG, Cantley LC, Thompson $\mathrm{CB}$. Understanding the Warburg effect: the metabolic requirements of cell proliferation. Science. 2009;324(5930):1029-1033.

25. Ju HQ, Zhan G, Huang A, et al. ITD mutation in FLT3 tyrosine kinase promotes Warburg effect and renders therapeutic sensitivity to glycolytic inhibition. Leukemia. 2017;31(10):2143-2150.

26. Poulain L, Sujobert P, Zylbersztejn F, et al High mTORC1 activity drives glycolysis addiction and sensitivity to G6PD inhibition in acute myeloid leukemia cells. Leukemia. 2017;31(11):2326-2335

27. Wang YH, Israelsen WJ, Lee D, et al. Cellstate-specific metabolic dependency in hematopoiesis and leukemogenesis. Cell. 2014;158(6):1309-1323.

28. Lagadinou ED, Sach A, Callahan $K$, et al BCL-2 inhibition targets oxidative phosphorylation and selectively eradicates quiescent human leukemia stem cells. Cell Stem Cell. 2013;12(3):329-341

29. Medeiros BC, Fathi AT, DiNardo CD, et al Isocitrate dehydrogenase mutations in myeloid malignancies. Leukemia. 2017; 31(2):272-281

30. Chan WK, Lorenzi PL, Anishkin A, et al. The glutaminase activity of L-asparaginase is not required for anticancer activity against ASNS-negative cells. Blood. 2014;123(23): 3596-3606.

31. Offman MN, Krol M, Patel N, et al. Rational engineering of $\mathrm{L}$-asparaginase reveals importance of dual activity for cancer cell toxicity. Blood. 2011;117(5):1614-1621.

32. Ando $M$, Sugimoto $K$, Kitoh $T$, et al Selective apoptosis of natural killer-cell tumours by l-asparaginase. Br J Haematol. 2005;130(6):860-868.

33. Willems L, Jacque $\mathrm{N}$, Jacquel $\mathrm{A}$, et al. Inhibiting glutamine uptake represents an attractive new strategy for treating acute myeloid leukemia. Blood. 2013; 122(20): 3521-3532.

34. Bertuccio SN, Serravalle S, Astolfi A, et al. Identification of a cytogenetic and molecular subgroup of acute myeloid leukemias showing sensitivity to L-Asparaginase. Oncotarget. 2017;8(66):109915-109923. 